\title{
Can giant radio halos probe the merging rate of galaxy clusters?
}

\author{
R. Cassano ${ }^{1}$, G. Brunetti ${ }^{1}$, C. Giocoli ${ }^{2}$, and S. Ettori ${ }^{3,4}$
}

\author{
1 INAF-Istituto di Radioastronomia, Bologna, via P.Gobetti, 101, 40129 Bologna, Italy \\ e-mail: rcassano@ira.inaf.it \\ 2 Aix Marseille Université, CNRS, LAM (Laboratoire d'Astrophysique de Marseille) UMR 7326, 13388 Marseille, France \\ 3 INAF/Osservatorio Astronomico di Bologna, via Ranzani 1, 40127 Bologna, Italy \\ 4 INFN, Sezione di Bologna, viale Berti Pichat 6/2, 40127 Bologna, Italy
}

Received 1 March 2016 / Accepted 13 June 2016

\begin{abstract}
Observations of galaxy clusters both in the radio and X-ray bands probe a direct link between cluster mergers and giant radio halos, suggesting that these sources can be used as probes of the cluster merging rate with cosmic time. While all giant radio halos are found in merging clusters, not every merging cluster hosts a giant radio halo. In this paper we carry out an explorative study that combines the observed fractions of merging clusters and radio halos with the merging rate predicted by cosmological simulations, and we attempt to infer constraints on merger properties of clusters that appear disturbed in X-rays and clusters that host radio halos. We used classical morphological parameters to identify merging systems and analysed the largest current (mass-selected $M_{500} \gtrsim 6 \times 10^{14} M_{\odot}$ and $0.2 \leq z \leq 0.33$ ) sample of galaxy clusters with radio and X-ray data; we extracted this sample from the Planck Sunyaev-Zeldovich cluster catalogue. We found that the fraction of merging clusters in this sample is $f_{\mathrm{m}} \sim 62-67 \%$, while that of clusters with radio halos is $f_{\mathrm{RH}} \sim 44-51 \%$. We assume that the morphological disturbance measured in the X-rays is driven by the merger with the largest mass ratio, $\xi\left(\xi=M_{i} / M_{1}<1\right.$, where $M_{i}$ and $M_{1}$ are the progenitor masses), which is still ongoing in the cluster at the epoch of observation. Results from theoretical studies allow us to derive the fraction of mergers with mass ratio above a minimum threshold (those with $\left.\xi \gtrsim \xi_{\min }\right)$ in our sample, under the assumption of a timescale $\tau_{\mathrm{m}}$ for the duration of merger-induced disturbance. The comparison of the theoretical merger fraction with the observed merger fraction allows us to constrain a region in the $\left(\xi_{\min }, \tau_{\mathrm{m}}\right)$ plane. We find that under the assumption of $\tau_{\mathrm{m}} \sim 2-3 \mathrm{~Gy}$, as constrained by simulations, the observed merger fraction matches the theoretical value for $\xi_{\min } \sim 0.1-0.18$. This is consistent with optical and near-infrared (IR) observations of galaxy clusters in the sample that constrain $\xi_{\min } \simeq 0.14-0.16$ through weak lensing analysis or study of the velocity distribution of galaxies in the clusters. The fact that radio halos are only found in a fraction of merging galaxy clusters may suggest that merger events generating radio halos are characterized by larger mass ratios; this seems to be supported by optical/near-IR observations of radio halo clusters in the sample that indeed allow us to constrain $\xi_{\min } \sim 0.2-0.25$. Alternatively, radio halos may be generated in all mergers but their lifetime is shorter (by $\sim f_{\mathrm{RH}} / f_{\mathrm{m}}$ ) than the timescale of the merger-induced disturbance. We stress that this is an explorative study, however it suggests that follow-up studies using the forthcoming radio surveys and adequate numerical simulations have the potential to derive quantitative constraints on the link between cluster merging rate and radio halos at different cosmic epochs and for different cluster masses.
\end{abstract}

Key words. galaxies: clusters: intracluster medium - cosmology: theory - radio continuum: general - X-rays: galaxies: clusters

\section{Introduction}

In the paradigm of the hierarchical structure formation scenario, galaxy clusters, the largest and more recently assembled structures in the Universe, form via mergers of smaller halos and continuous accretion of unbound matter. The process of mass accretion of dark matter halos is a clear outcome of the cosmological model. This process can be statistically investigated with $N$-body simulations and semi-analytical models through the identification of merger trees of dark matter halos, which lead to the derivation of the mass accretion history and merging rate as a function of redshift, halo mass, and mass ratio of the progenitors (e.g. van den Bosh 2002; Giocoli et al. 2007; Moreno et al. 2007; 2008; Fakhouri \& Ma 2008; McBride et al. 2009; Fakhouri et al. 2010; Giocoli et al. 2012).

Observationally, the exploration of the merging rate of dark matter halos has only been attempted on the scales of galaxies using two main methods for tracing the merging history in the observations: morphological identification techniques (Conselice et al. 2003; Lotz et al. 2004) and the close galaxy pair method (e.g. Patton et al. 2000; De Propris et al. 2005). These methods are then combined with the merger timescale derived from $N$-body simulations to obtain the merging rate (e.g. Lotz et al. 2011; Jian et al. 2012; Conselice 2014). Current results are inconclusive, however, because the merger rate of dark matter halos and the merger rate of galaxies do not necessary coincide. This is because they are related by dissipative processes, such as dynamical friction, tidal interaction, and stellar feedback, that are difficult to model (Fakhouri \& Ma 2008; Guo \& White 2008; Lotz et al. 2011; Hopkins et al. 2013). Dissipative processes are instead less relevant during the mass accretion of galaxy clusters. Nevertheless only recently a method based on the possibility to measure the cluster mass in a thin spherical shell surrounding the cluster beyond $R_{200}$ (with the caustic technique) and by estimating its infalling time (e.g. Diaferio 2015; De Boni et al. 2016), has been proposed to measure the mass accretion rate of galaxy clusters. Yet, in general, the growth of structures on the scale of galaxy clusters remains poorly explored from an observational prospective (e.g. Lemze et al. 2013).

Mergers between clusters are the most energetic phenomena since the Big Bang with a release of a gravitational potential energy of $\sim 10^{63}-10^{64}$ erg during one cluster crossing time 
( $\sim 1$ Gyr). During such events, shock waves and random vortical flows, if not turbulence, are produced in the intracluster medium (ICM; e.g. Kulsrud et al. 1997; Norman \& Bryan 1999; Ricker $\&$ Sarazin 2001). These motions originate as a result of vorticity generation in oblique accretion shocks and instabilities during the cluster formation, and in the wake of the smaller subclusters (e.g. Subramanian et al. 2006; Brunetti \& Jones 2014; Brüggen $\&$ Vazza 2015). The bulk of the gravitational energy associated with the collision is released as thermal energy in the final system (e.g. Kravtsov \& Borgani 2012), while another fraction can be channelled into non-thermal plasma components, i.e. relativistic particles and magnetic fields in the ICM (e.g. Brunetti $\&$ Jones 2014). The existence of cosmic ray electrons and magnetic fields in the ICM is in fact demonstrated by radio observations. Cluster-scale ( $\sim \mathrm{Mpc}$-scale) diffuse synchrotron emission is frequently found in merging galaxy clusters in the form of so-called giant radio halos (hereafter RHs or RH), apparently unpolarized synchrotron emission associated with the cluster $\mathrm{X}$-ray emitting regions, and giant radio relics, elongated and often highly polarized synchrotron sources typically seen in the clusters outskirts (e.g. Feretti et al. 2012, for an observational review). The properties of radio relics suggest a connection with large-scale shocks that cross the ICM during mergers and may accelerate locally injected electrons or reaccelerate pre-existing energetic electrons, while RHs likely trace gigantic turbulent regions in the ICM, where relativistic electrons can be reaccelerated through scattering with magnetohydrodynamic (MHD) turbulence (e.g. Brunetti \& Lazarian 2007; Brüggen et al. 2012).

In recent decades, radio observations of statistical samples of galaxy clusters have shown that RHs are not ubiquitous; only $\sim 20-30 \%$ of the X-ray luminous $\left(L_{X}(0.1-2.4 \mathrm{keV}) \geq\right.$ $5 \times 10^{44} \mathrm{erg} / \mathrm{s}$ ) clusters host a RH (e.g. Venturi et al. 2008; Kale et al. 2015), while the fraction of clusters with RHs becomes larger in Sunyaev Zeldovich (SZ)-selected clusters (e.g. Basu 2012; Cassano et al. 2013; Sommer \& Basu 2014; Cuciti et al. 2015; Bernardi et al. 2016). Most importantly, it was found that RH and non-RH clusters are clearly separated in the $P_{1.4}-L_{\mathrm{X}}$ and $P_{1.4}-M_{500}\left(Y_{500}\right)$ diagrams according to the cluster dynamical status, where RHs are always associated with dynamically disturbed clusters and clusters without RHs are statistically more relaxed (e.g. Brunetti et al. 2007, 2009; Cassano et al. 2010, 2013). The connection between RHs and merging clusters has been further supported by a number of independent studies (e.g. Rossetti et al. 2011; Wen \& Han 2013; Parekh et al. 2015; Mantz et al. 2015; Yuan et al. 2015; Kale \& Parekh 2016). The RH-cluster merger connection suggests that RHs can be used as signposts of cluster mergers and supports the idea that RHs are transient phenomena tracing turbulent regions in the ICM during the process of cluster formation. Not all merging clusters host a giant RH (see Cassano et al. 2013 and ref. therein), however, and this poses fundamental questions about the conditions that are necessary to generate cluster-scale synchrotron diffuse emission.

The connection between mergers and non-thermal phenomena also opens to the possibility of inferring constraints on the cluster merging rate from radio observations. In this paper we start exploring this possibility. In particular we attempt to combine the observed fraction of merging clusters and the observed fraction of RHs in clusters with the merging rate predicted by cosmological simulations to infer constraints on the properties of the mergers that induce disturbances observed in X-rays and those responsible for RHs.

We stress that this is an explorative study whose the main aim is to start to investigate the possibility to use diffuse radio emission in galaxy clusters as tracer of the cluster dynamical status. In particular, we stress that current statistical information is still limited to very massive $\left(M_{500} \gtrsim 6 \times 10^{14} M_{\odot}\right)$ and relatively nearby systems $(z \simeq 0.2-0.33)$, while we anticipate that better constraints can be obtained using less massive systems or clusters at higher redshifts.

In Sect. 2 we present the cluster sample and derive the fractions of merging clusters and those of clusters with RHs; in Sect. 3 we describe the formalism by Fakhouri et al. (2010) to derive the merging rate in simulations and derive the expected merger fraction. In Sect. 4 we compare the observed merger fraction and $\mathrm{RH}$ fraction with expectations from simulations, and we attempt to constrain the properties that cluster mergers should have to explain the observed fraction of clusters with X-ray disturbances and that of clusters with RHs. Finally, in Sect. 5 we summarize the main results and discuss the main implications for the origin of giant RHs in galaxy clusters.

A Lambda cold dark matter $(\Lambda \mathrm{CDM})$ cosmology $\left(H_{0}=\right.$ $\left.70 \mathrm{~km} \mathrm{~s}^{-1} \mathrm{Mpc}^{-1}, \Omega_{\mathrm{m}}=0.3, \Omega_{\Lambda}=0.7\right)$ is adopted.

\section{Data and sample selection}

We used the Planck SZ cluster catalogue (PSZ; Planck Collaboration XXIX 2014) to select 54 clusters with $M_{500} \gtrsim 6 \times$ $10^{14} M_{\odot}{ }^{1}$, redshift $0.2 \leq z \leq 0.33$ and $\delta>-30^{\circ}$ and $|b| \geq \pm 20^{\circ}$, where $b$ is the galactic latitude (Table A.1). With such a selection the sample has a mass completeness of $\sim 80 \%{ }^{2}$.

This selection has been thought to optimize the available information in the radio band; indeed 37 out of 54 clusters belong to the Giant Metrewave Radio Telescope (GMRT) RH Survey and its extension (EGRHS; Venturi et al. 2007, 2008; Kale et al. $2013,2015)$ and for 39 out of 54 clusters ( $72 \%$ of the sample) information about the presence/absence of diffuse radio emission is available. In particular, 17 clusters host giant RHs, while 3 clusters host candidate RHs (see Table A.1). The fraction of RHs, defined as $f_{\mathrm{RH}}=N_{\mathrm{H}} / N_{\text {tot }}$, where $N_{\mathrm{H}}$ is the number of RHs and $N_{\text {tot }}$ the total number of clusters, is thus $\sim 44 \%$ and can reach $\sim 51 \%$ if we include the 3 uncertain cases.

Fifty-one out of 54 clusters ( $~ 94 \%$ of the sample, including all the 39 clusters with available radio information) have X-ray data (Chandra and/or XMM-Newton) that can be used to derive information about the cluster dynamical status. In particular, 41 of these clusters have Chandra data for which morphological indicators, such as the power ratio $P_{3} / P_{0}$ (e.g. Buote \& Tsai 1995), the emission centroid shift $w$ (e.g. Mohr et al. 1993), and the surface brightness concentration parameter $c$ (e.g. Santos et al. 2008), can be homogeneously derived to quantitatively establish the cluster dynamical status. Following Cassano et al. (2010, 2013), we adopted an algorithm for an automatic detection of the point sources, which are then removed from the images. We study the cluster substructures on a typical RH scale by analysing the surface brightness inside an aperture radius of $500 \mathrm{kpc}$, since we are interested in the cluster dynamics on the scales where the energy is most likely dissipated to generate radio emission. The power ratio is a multipole decomposition of the two-dimensional projected mass distribution within a given aperture, and $P_{3} / P_{0}$ is the lowest power ratio moment providing a clear substructure measure (Böhringer et al. 2010). The centroid shift $w$ is defined as the standard deviation of the projected

The values of $M_{500}$ in the PSZ catalogue are obtained from $Y_{500}$ as described in Sect. 7.2.2 in Planck Collaboration XXIX (2014).

2 This completeness is estimated in $Y_{S Z}$ by Planck Collaboration XXIX (2014) and then converted in "mass completeness" using scaling relations in Planck Collaboration XX (2014; see Fig. 28 in Planck Collaboration XXIX 2014). 
Table 1. Cluster fractions.

\begin{tabular}{ccc}
\hline \hline Cluster sample & $f_{\mathrm{m}}$ & $f_{\mathrm{RH}}$ \\
\hline 39 clusters & $62-67 \%$ & $44-51 \%$ \\
51 clusters & $65-69 \%$ & $45-51 \%$ \\
\hline
\end{tabular}

separation between the peak and centroid of the cluster X-ray brightness distribution in unit of the aperture radius. The concentration parameter $c$ is defined as the ratio of the peak (within $100 \mathrm{kpc}$ ) over the ambient (within $500 \mathrm{kpc}$ ) X-ray surface brightness. Following previous papers (Cassano et al. 2010, 2013; Cuciti et al. 2015), we adopt the following threshold values to classify clusters as mergers: $P_{3} / P_{0} \gtrsim 1.2 \times 10^{-7}, w \gtrsim 0.012$ and $c \lesssim 0.2$. Morphological parameters are already published in Cassano et al. (2010, 2013) and Cuciti et al. (2015) for 32 clusters with Chandra data; here we derive the morphological quantities for additional 9 clusters following the approach outlined above (and described in previous works; see e.g. Sect. 3 of Cassano et al. 2010, for details). The resulting dynamical status of the clusters, "merger" versus "relaxed", is reported in Table A.1 (Col. 6); the values of the morphological parameters for the 41 clusters with Chandra data are reported in Table A.2.

Ten more clusters with available XMM-Newton observations can be added to this sample and after a visual inspection of their images we can assess (even if with less confidence) their dynamical status (also reported in Table A.1, Col. 6).

In deriving the merger fraction ${ }^{3}$ we assume that the disturbance we measure in the X-rays is mainly due to the merger with the largest mass ratio that is ongoing in the system at the epoch of the observation, i.e. a binary merger approximation. Under this assumption the merger fraction is equivalent to the fraction of clusters that is actually in phase of merger (where we consider clusters as the final product of the merger). Considering the sample of 39 clusters with available radio information, we found that the fraction of dynamically disturbed systems, or the merger fraction, which is defined as $f_{\mathrm{m}}=N_{\mathrm{m}} / N_{\text {tot }}$ with $N_{\mathrm{m}}$ being the number of merging clusters, is $\sim 62-67 \%$ (including the uncertainty on the classification of two clusters; see Table A.1). If we extend this analysis to the sample of 51 clusters with X-ray data, we found $f_{\mathrm{m}} \sim 65-69 \%$. We can only speculate on the fraction of RHs in this sample of 51 clusters, for instance, by assuming that the fraction of merging clusters with RHs in these additional 12 clusters is the same as we measure in the sample of 39 clusters (that is $\sim 70 \%$ ); we obtain $f_{\mathrm{H}} \sim 45-51 \%$. The derived fractions are summarized in Table 1.

Calculations in the paper are based on the fractions extracted from the sample of 39 clusters (for which both radio and X-ray data $^{4}$ are available), although these fractions are not expected to change significantly in the extended sample (under reliable assumptions, see Table 1).

\section{Merging rate of halos from simulations}

In the $\Lambda \mathrm{CDM}$ scenario dark matter halos grow in mass and size primarily through mergers with other halos: merger with com-

\footnotetext{
3 To compare the observed merger fraction with theoretical expectations, we convert the $M_{500}$ to virial masses, $M_{\mathrm{vir}}$, by assuming a NFW profile (e.g. Navarro et al. 1997) for the dark matter halos and the concentration-mass relation in Duffy et al. (2008); see Appendix A in Ettori et al. (2010). Both values of $M_{500}$ and $M_{\text {vir }}$ are reported in Table A.1.

4 For 4 out of 39 clusters, the dynamical classification is based on a visual inspection of the XMM-Newton cluster image.
}

parable mass halos ("major mergers") and merger with smaller satellite halos ("minor mergers"). To derive the merging rate of halos we use the result derived from the combined Millennium (Springel et al. 2005) and Millennium-II (Boylan-Kolchin et al. 2009) simulations (Fakhouri et al. 2010; FMB10 hereafter). FMB10 used merger trees of dark matter halos to extract a catalogue of mergers containing for each descendent halo at redshift $z_{\mathrm{d}} \geq 0$ with mass $M$ the $N_{\mathrm{p}}\left(N_{\mathrm{p}} \geq 1\right)$ progenitors at $z_{\mathrm{p}}=z_{\mathrm{d}}+\Delta z$, with masses $M_{1} \geq M_{2} \geq \ldots M_{N_{\mathrm{p}}}$. These authors include all the progenitors (above a given mass threshold) to derive the merging rate, and since they do not have information about the order the progenitors merge, they assume that each progenitor $M_{i}$ with $i \geq 2$ mergers with the most massive progenitor $M_{1}$ at a given point between $z_{\mathrm{p}}$ and $z_{\mathrm{d}}$. Thus a descendent halo with $N_{\mathrm{p}}$ progenitors is assumed to be the result of $N_{\mathrm{p}}-1$ binary merger events with mass ratio $\xi=M_{i} / M_{1} \leq 1\left(i=2, \ldots, N_{\mathrm{p}}\right)$. The progenitor mass ratio, $\xi$, is defined so that, for instance, $\xi=0.3$ indicates major mergers with mass ratio $1: 3$, while $\xi=0.1$ indicates mergers with mass ratio $1: 10$. The derived mean merging rate per halo, $\mathrm{d} N_{\mathrm{m}} / \mathrm{d} \xi / \mathrm{d} z$, which gives the mean number of mergers per unit halo per unit $z$ per unit $\xi$, can be well described by the following formula (FMB10):

$\frac{\mathrm{d} N_{\mathrm{m}}}{\mathrm{d} \xi \mathrm{d} z}(M, \xi, z)=A\left(\frac{M}{10^{12} M_{\odot}}\right)^{\alpha} \xi^{\beta} \exp \left[\left(\frac{\xi}{\tilde{\xi}}\right)^{\gamma}\right](1+z)^{\eta}$,

were the best-fit parameters are: $\alpha=0.133, \beta=-1.995, \gamma=$ $0.263, \eta=0.0993$, and $A=0.0104, \tilde{\xi}=9.72 \times 10^{-3}$. This formula has a negligible dependence on the redshift and it is also nearly independent of the mass. The main dependence is on the mass ratio $\xi$, so that the number of mergers per halo is larger for smaller mass ratio. For example, the number of mergers (per halo) with $\xi=0.01$ is about 90 times larger than the number of mergers with $\xi=0.1$ (see Fig. 1, right panel, in FMB10).

Integrating Eq. (1) for $\xi \geq \xi_{\min }$ and between $z_{0}$ and $z$, one obtains the cumulative number of mergers, $N_{\mathrm{m}}\left(\xi_{\min }, M_{0}, z_{0}, z\right)$. That is the total number of mergers with $\xi \geq \xi_{\min }$ that a halo of mass $M_{0}$ at redshift $z_{0}$ has encountered between $z_{0}$ and an earlier $z$ during the halo's history,

$N_{\mathrm{m}}\left(\xi_{\min }, M_{0}, z_{0}, z\right)=\int_{z_{0}}^{z} \mathrm{~d} z \int_{\xi_{\min }}^{1} \mathrm{~d} \xi \frac{\mathrm{d} N_{\mathrm{m}}}{\mathrm{d} \xi \mathrm{d} z}(M(z), \xi, z)$,

where $M(z)$ is the mass accretion history and can be obtained integrating the fitting formula for the mass accretion rate, $\dot{M}=$ $\left(M_{0}-M_{1}\right) / \Delta_{t}$, where $M_{0}$ is the descendent mass at time $t$ and $M_{1}$ is the mass of its most massive progenitor at time $t-\Delta_{t}$, that is given by (FMB10)

$\langle\dot{M}\rangle_{\text {mean }}=46.1 M_{\odot} \mathrm{yr}^{-1}\left(\frac{M}{10^{12} M_{\odot}}\right)^{1.1} \times E_{z}(1+1.11 z)$.

The quantity we derived observationally is the merger fraction, which is the fraction of clusters with significant dynamical disturbance in X-rays (see Sect. 2). To compare this quantity with expectations given by Eqs. (1)-(3), we need to assume a merger timescale, $\tau_{\mathrm{m}}$, associated with the duration of the morphological disturbance that we infer from X-ray images. This is a free parameter in our calculations that however can be constrained through numerical simulations (see Sect. 4). We derive the average fraction of mergers with $\xi \geq \xi_{\min }$ expected in our sample by integrating Eq. (2) for each cluster of the sample with mass $M_{0}$ 


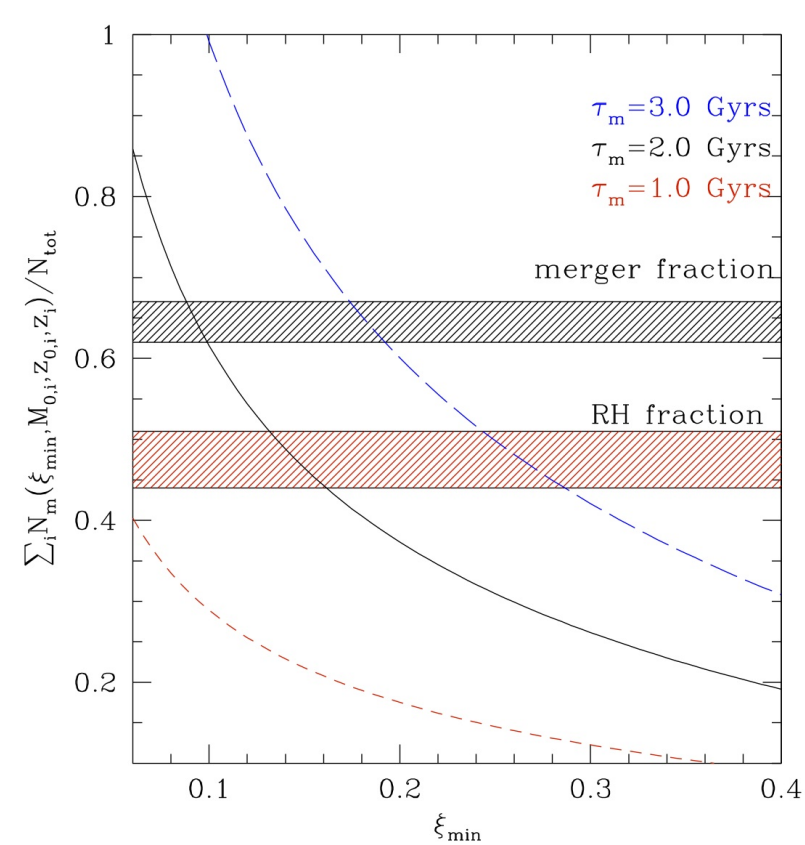

Fig. 1. Predicted average merger fraction for clusters in the sample as a function of $\xi_{\min }$, assuming three different values for the merger timescale $\tau_{\mathrm{m}}=1,2,3 \mathrm{Gyr}$ (from bottom to top). The observed merger fraction and RH fraction are also reported (shadowed regions).

and redshift $z_{0}$ up to the redshift $z$ corresponding to the lookback time $\tau_{\mathrm{m}}$ and then computing $\sum_{i} N_{\mathrm{m}}\left(\xi_{\mathrm{min}}, M_{0, i}, z_{0, i}, z_{i}\right) / N_{\text {tot }}$, where the sum is on the $N_{\text {tot }}$ number of clusters in the sample. The derived average merger fraction is reported in Fig. 1 as a function of $\xi_{\min }$ for three values of $\tau_{\mathrm{m}}\left(\tau_{\mathrm{m}}=1,2,3 \mathrm{Gyr}\right)$ and is compared with the observed merger fraction and observed fraction of clusters with RH (shadowed regions).

The predicted merger fraction decreases for larger mass ratios simply because major mergers are less common than minor mergers and it obviously increases by assuming larger timescales.

\section{Comparison with theory}

The comparison between the observed and theoretical merging fractions allows us to derive constraints on relevant parameters, such as $\tau_{\mathrm{m}}$ and $\xi_{\mathrm{min}}$. Before proceeding in this direction, we need to discuss some caveats in our procedures. In principle, the comparison between the "observed" and "theoretical" merging fractions allows us to derive constraints on relevant parameters, such as $\tau_{\mathrm{m}}$ and $\xi_{\min }$.

\subsection{Caveats}

The observed merger fraction is derived by measuring the fraction of clusters with significant X-ray disturbances. This means that our method is limited to events with significant mass accretion, otherwise it would be difficult to classify these events as "mergers" based on the morphological parameters. The dynamical parameters are derived within a region of radius $500 \mathrm{kpc}$. On the other hand, in FMB10 the merger fraction is derived from the merging rate that considers all the infalling halos within the virial radius of the main cluster (that for our clusters is $\sim 2-3 \mathrm{Mpc}$ ). Since we are considering a "rate", or number of infalling halos per unit time, it is important that the halos crossing the virial radius of the main cluster cross, at a given time, the radius of

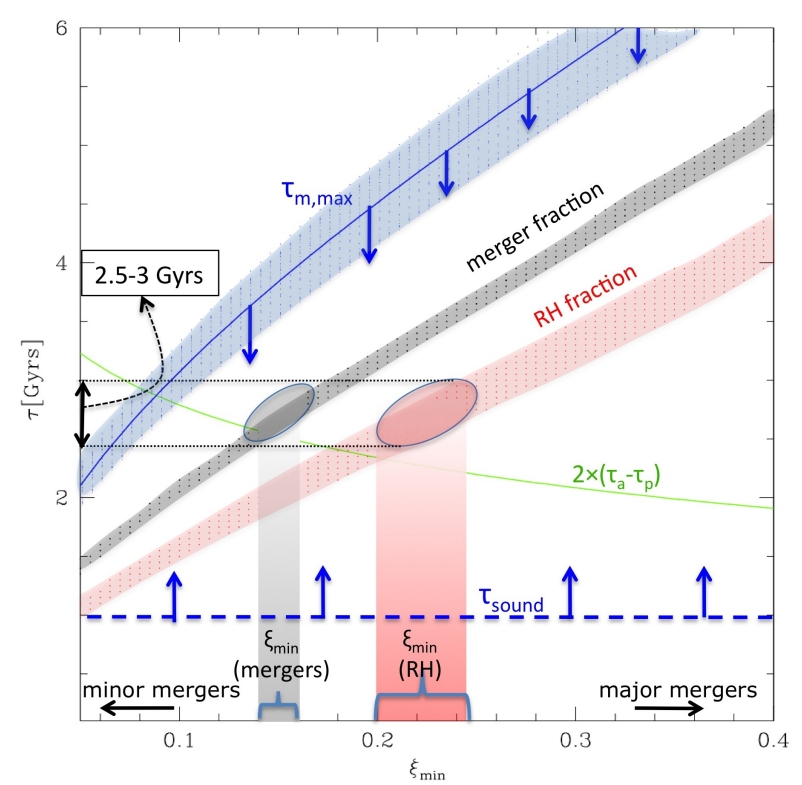

Fig. 2. Allowed regions of parameters $\left(\xi_{\min }, \tau_{\mathrm{m}}\right)$ constrained by requiring that the observed merger fraction (black region) and $\mathrm{RH}$ fraction (red regions) match those predicted by theory (these regions account for the uncertainty in the observed fraction). For each $\xi_{\min }$ the maximum allowed value of $\tau_{\mathrm{m}}, \tau_{\mathrm{m} \text {,max }}$ (blue region with arrows; see Sect. 4.2) and a lower limit to $\tau_{\mathrm{m}}$ (horizontal blue dashed line, see Sect. 4.2) are also shown. The merger timescale constrained by cosmological simulation is also reported (green line; Tormen et al. 2004). Ranges of $\xi_{\min }$ constrained through optical/near-IR observations of galaxy clusters in the sample (see Sect.4.4) are shown for merging clusters (black rectangular region ) and for clusters with $\mathrm{RHs}$ (red rectangular region). These values of $\xi_{\text {min }}$ both constrain $\tau_{\mathrm{m}} \sim \tau_{\mathrm{RH}} \sim 2.5-3 \mathrm{Gyr}$; this is also show in the figure.

$500 \mathrm{kpc}$ reaching the central regions. According to cosmological simulations, halos with a mass ratio $\xi \geq 0.1$ reach their pericentric distance, that is $\sim 0.2-0.3 R_{v}$, within a timescale of $\sim 0.9 \mathrm{Gyr}$ from the virial crossing (e.g. Tormen et al. 2004).

The other assumption is that of binary mergers, i.e. we assume that the disturbance we measure in the X-rays is mainly caused by a binary merger event between the two main progenitors. Consequently, we derive the expected average merger fraction from the fitting formulae by FMB10 assuming that in a merger timescale there is a main binary merger event with $\xi \gtrsim \xi_{\min }$ that influences the observed dynamical status. However, in a merger timescale, especially for a long timescale, clusters might experience multiple merger episodes characterized by lower mass ratio $\left(\xi<\xi_{\min }\right)$. If the number of mergers with a mass ratio that is slightly lower than $\xi_{\min }$ is significant, our assumption would be no longer valid since the interplay of these mergers would also contribute to the morphological disturbance.

\subsection{Mergers and observed X-ray morphology}

Given these premises, we proceed with the comparison between the observed merger fraction and the results by FMB10. From Fig. 1 it is clear that we can find combinations $\left(\tau_{\mathrm{m}}, \xi_{\min }\right)$ for which the expected merger fraction can match that observed in our sample. Figure 2 (black region) shows the allowed regions of $\left(\tau_{\mathrm{m}}, \xi_{\mathrm{min}}\right)$ that is derived by matching theory and observations. As expected, there is a clear degeneracy between $\tau_{\mathrm{m}}$ and $\xi_{\min }$.

We first identify forbidden regions in the $\tau_{\mathrm{m}}-\xi_{\text {min }}$ diagram. A lower limit on $\tau_{\mathrm{m}}$ can be obtained by assuming that the 


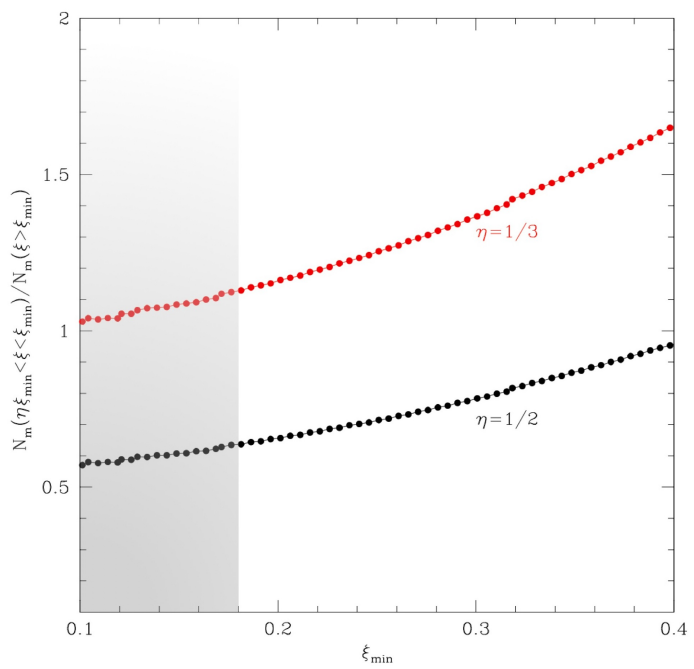

Fig. 3. Ratio between the number of mergers with $\eta \xi_{\text {min }}<\xi<\xi_{\text {min }}$ and that with $\xi \geq \xi_{\min }$ for $\eta=1 / 2$ (bottom black dots) and $\eta=1 / 3$ (upper red dots). The shadowed region indicates the range of $\xi_{\min }=$ 0.1-0.18 constrained by the merger timescale derived from numerical simulations (Tormen et al. 2004).

merger-driven perturbations within a region of diameter $1 \mathrm{Mpc}$ cannot last for a timescale shorter than the sound crossing time of that region, which for a galaxy cluster with $T \sim 10^{8} \mathrm{~K}$ is $\tau_{\text {sound }} \simeq 1$ Gyr. The values of $\tau_{\mathrm{m}}$ constrained by the observations are always larger than $\tau_{\text {sound }}$ (at least for $\xi_{\text {min }} \geq 0.05$ ). An upper bound to $\tau_{\mathrm{m}}$ can be derived by considering the fact that extremely large duration of mergers would make dynamically disturbed all the clusters that are observed at a given cosmic epoch. Specifically, for each cluster of the sample with mass $M_{0}$ at redshift $z_{0}$ we derive the values of $\tau_{\mathrm{m} \text {,max }}$, as a function of the progenitor mass ratio $\xi_{\min }$, corresponding to the redshift $z$ for which Eq. (2) gives $N_{\mathrm{m}}=1$. This means that a merger event with $\xi \geq \xi_{\min }$ is still producing a disturbance in all clusters of our sample at the epoch of observation. The derived distribution of $\tau_{\mathrm{m}, \max }$, for each value of $\xi_{\min }$, and its mean value are reported in Fig. 2 (blue region and line, respectively); the values of $\tau_{\mathrm{m}}$ constrained by the observations are always smaller than $\tau_{\mathrm{m} \text {,max }}$.

Owing to the degeneracy between $\tau_{\mathrm{m}}$ and $\xi_{\min }$ in principle large merger timescales can be admitted to explain the observed merger fraction. As already anticipated, however, under this condition our hypothesis of binary mergers can be no longer valid since multiple merger events with mass ratio slightly smaller than $\xi_{\min }$ can contribute to the disturbance. To check this, hydrodynamical simulations are necessary to unambiguously relate the merger mass ratio to the cluster morphological parameters, but this is beyond the scope of the paper and deserves future ad hoc simulations. Here we limit ourselves to the following test. In Fig. 3 we use the values $\left(\tau_{\mathrm{m}}, \xi_{\mathrm{min}}\right)$ constrained in Fig. 2 and show the ratio between the number of mergers with mass ratio in the range $\eta \xi_{\min }-\xi_{\min }$ and that of mergers with $\xi \geq \xi_{\min } ; \eta=1 / 2$ and $1 / 3$ are considered. We conclude that the binary approach adopted in our paper is appropriate for merger timescale as large as 3-4 Gyr (those corresponding to $\xi_{\min } \sim 0.2-0.3$ ).

A possibility to break the degeneracy between $\tau_{\mathrm{m}}$ and $\xi_{\text {min }}$ is to adopt values of $\tau_{\mathrm{m}}$ inferred from numerical simulations. A reference timescale is the time necessary for a subcluster to complete an orbit around the centre of mass of the main cluster. Following Tormen et al. (2004) this time can be estimated as $2 \times\left(\tau_{\mathrm{a}}-\tau_{\mathrm{p}}\right)$, where $\tau_{\mathrm{a}}=\tau_{\mathrm{a}}(\xi)=1.6(\xi+0.02)^{-0.17} \mathrm{Gyr}$ and $\tau_{\mathrm{p}}=0.9$ Gyr are the apocentric and pericentric timescale, re- spectively. This timescale is reported in Fig. 2 (green line) as a function of $\xi_{\mathrm{min}}$. It intercepts the region constrained by the observations for $\tau_{\mathrm{m}} \simeq 2.5 \mathrm{Gyr}$ implying $\xi_{\min } \simeq 0.14$. In Tormen et al. (2004), $\tau_{\mathrm{a}}$ and $\tau_{\mathrm{p}}$ are derived from analytic fits to the results of numerical simulations, however the dispersion around the median value is $\sim 0.5 \mathrm{Gyr}$. As a consequence the merger timescale is constrained as $\tau_{\mathrm{m}} \sim 2-3 \mathrm{Gyr}$, implying $\xi_{\text {min }} \sim 0.1-0.18$. Coming back to Fig. 3, we note that for $\xi_{\min } \simeq 0.1-0.18$ (shadowed region) the number of mergers with mass ratio $1 / 3 \xi_{\min }<\xi<$ $\xi_{\min }$ is about the same as that of mergers with $\xi \geq \xi_{\min }$ and that the number of mergers with $1 / 2 \xi_{\min }<\xi<\xi_{\min }$ is about half of that of mergers with $\xi \geq \xi_{\min }$. As these mergers are characterized by relatively small mass ratio $(\xi \sim 0.03-0.09)$ and are not numerous, they should have a negligible influence on the cluster morphological parameters, and thus our assumption of binary mergers is reasonably correct.

\subsection{Mergers and radio halos}

Radio halos are always observed in dynamically disturbed systems, however they are found only in a fraction of the clusters that are classified as merging systems. In this section we follow the approach already adopted in Sect. 4.2 and attempt to constrain the properties that cluster mergers should have to explain the observed fraction of clusters with RHs. In Fig. 2 we report on the region $\left(\tau_{\mathrm{m}}, \xi_{\mathrm{min}}\right)$ constrained by requiring that the predicted merger fraction matches the observed fraction of clusters with RHs (red shadowed region). At this point we can adopt two main scenarios:

(i) We can assume that the lifetime of a $\mathrm{RH}$ is equivalent to the lifetime of the merger-induced disturbances identified by X-ray parameters. Under this hypothesis and since RHs are found in disturbed systems, we can assume that RHs are generated in those systems that have larger mass ratios among merging clusters in our sample. In this case we derive $\xi_{\min } \sim 0.18-0.25$ for $\tau_{\mathrm{m}}=2-3 \mathrm{Gyr}$ as constrained in Sect. 4.3.

(ii) We can assume that RHs are statistically generated in all mergers identified in our sample, but they are short-lived compared to the timescale of the merger-induced disturbance. Under this assumption, the lifetime of a RH is simply $\tau_{\mathrm{RH}} \sim \tau_{\mathrm{m}} \times\left(f_{\mathrm{RH}} / f_{\mathrm{m}}\right) \sim(0.7-0.8) \tau_{\mathrm{m}}$, i.e. $\tau_{\mathrm{RH}} \sim 1.4-2.4 \mathrm{Gyr}$ for $\tau_{\mathrm{m}}=2-3 \mathrm{Gyr}$.

\subsection{Constraints on $\xi_{\min }$ from observations}

The mass ratio $\xi_{\min }$ is a simple outcome of our procedure that is appropriate for binary mergers, however, this value can be constrained independently by observations of clusters in our sample. Observations of single clusters may be used to derive independent constraints on $\xi$. We collected information in the literature about the mass ratio of the merging clusters of our sample. These mass ratio are derived from optical or near-IR observations of galaxy clusters through weak lensing analysis or through the study of the galaxy velocity distributions in the clusters (see Table A.1). We found information for seven clusters with RH and for Z5247, which hosts a candidate RH (see Table A.1). For five merging clusters without RH, we looked at the reconstructed convergence maps from weak gravitational lensing (see Table A.1). For these clusters we estimated the mass ratio of the different merging components adopting circular filters on the reconstructed convergence maps with a typical scale that allows us to isolate the different correlated peaks. Owing to the restricted 
number density of sources beyond the clusters from which the weak lensing signal is measured and and also to the limited resolution of the recovered convergence maps (around the arcmin scale), we stress that in these cases the quoted values represent an upper limit for the mass ratio.

The minimum values of $\xi$ we found for merging clusters in our sample is $\sim 0.14-0.16$. If we use these values, $\xi_{\text {min }} \sim$ 0.14-0.16 (black rectangular region in Fig. 2), we can derive $\tau_{\mathrm{m}} \simeq 2.5-3 \mathrm{Gyr}$, which is consistent with the reference values of merging timescales derived from results of cosmological simulations (see Fig. 2).

We found some evidence (the information is available only for half of the merging clusters in the sample) that merging clusters with $\mathrm{RH}$ are in general characterized by mergers with larger mass ratio than merging clusters without $\mathrm{RH}$ : $\xi$ ranges from $\xi \sim 0.2-0.25$ up to $\xi \sim 1$. If we assume $\xi_{\text {min }} \sim 0.2-0.25$ for merging clusters with $\mathrm{RH}$ (red rectangular region in Fig. 2), we constrain $\tau_{\mathrm{RH}} \sim \tau_{\mathrm{m}} \sim 2.5-3 \mathrm{Gyr}$, thus in this case the RH lifetime would be comparable with the merger timescale, potentially supporting the scenario (i) in Sect. 4.3.

\section{Summary and discussion}

Observations establish a clear connection between RHs and mergers (e.g. Cassano et al. 2010), suggesting that RHs can be used as probes of the cluster merging rate with cosmic time. Based on this possibility, in this paper we carry out an exploratory study. By combining the observed fraction of merging clusters and the observed fraction of $\mathrm{RH}$ in clusters with the merging rate predicted by fitting formulae based on cosmological simulations, we attempt to infer constraints on merger properties of clusters that appear disturbed in X-rays and of clusters that host RHs.

We use the Planck SZ cluster catalogue (PSZ; Planck Collaboration XXIX 2014) and select a sample of 54 clusters with mass $M_{500} \gtrsim 6 \times 10^{14} M_{\odot}$ and redshift $0.2 \leq z \leq 0.33$. 39 of these clusters have both X-ray and radio information and represent a subsample that can be used to measure the fraction of RHs and that of merging clusters. Mergers in the sample are identified by means of X-ray morphological parameters. We find that all RHs are in merging clusters whereas not all merging clusters host a RH; specifically $\sim 44-51 \%$ of the clusters in the sample have a RH, while (using Chandra and XMM-Newton X-ray data) the total fraction of merging clusters is $\sim 62-67 \%$.

We convert the theoretical merging rate per halo (FMB10), which mainly depends on the mass ratio of the two progenitors, $\xi=M_{i} / M_{1}<1$, into merger fraction by adopting a merger timescale $\tau_{\mathrm{m}}$ as a free parameter. The predicted fraction of merging clusters has a strong dependence on $\xi_{\min }$, i.e. the minimum mass ratio of the mergers (larger is $\xi_{\min }$ smaller is the number of mergers; Fig. 1) and on $\tau_{\mathrm{m}}$ (larger is $\tau_{\mathrm{m}}$ larger is the expected fraction of merging clusters; Fig. 1). The comparison between the observed and predicted merger fraction allows us to constrain an allowed region in the diagram $\left(\xi_{\min }, \tau_{\mathrm{m}}\right)$ where there is degeneracy between these two parameters (Fig. 2).

We attempt to break the degeneracy between $\xi_{\min }$ and $\tau_{\mathrm{m}}$ :

a) by assuming the merger timescale that is derived by cosmological simulations (e.g. Tormen et al. 2004), $\tau_{\mathrm{m}} \sim 2-3 \mathrm{Gyr}$, we find that a value $\xi_{\min } \sim 0.1-0.18$ explains the observed merger fraction;

b) by assuming values of $\xi_{\min }$ derived through the analysis of optical or near-IR observations of merging clusters in the sample $\xi_{\min } \sim 0.14-0.16$, we find that a merger timescale $\tau_{\mathrm{m}} \simeq 2.5-3$ Gyr explains the observed merger fraction.

Interestingly, values of the parameters that are obtained independently in a) and b) are consistent.

We find that all clusters with RH in our sample are merging systems but that not all merging clusters host a RH. There are two main possibility to interpret this difference:

- Scenario 1) RHs have lifetimes similar to the lifetimes of merger-driven disturbances in the X-rays, but they are generated in the merging events with larger mass ratios. Values of mass ratios derived from optical or near-IR observations of galaxy clusters in the sample (through weak lensing analysis or through the study of the velocity distribution of galaxies in the clusters) may support this possibility. Indeed we find that $\xi$ ranges between $\sim 0.2-0.25$ and $\sim 1$ for RH clusters, whereas values of $\xi \sim 0.14-0.16$ up to $\xi \sim 0.25-0.3$ are found for merging clusters without RH. If we assume $\xi_{\min } \sim 0.2-0.25$, we find that $\tau_{\mathrm{RH}} \sim \tau_{\mathrm{m}} \simeq 2-2.5$ Gyr should be adopted to explain the observed $\mathrm{RH}$ fraction.

- Scenario 2) the lifetime of RHs $\left(\tau_{\mathrm{RH}}\right)$ is shorter than the timescale of merger-induced disturbance in the X-rays with $\tau_{\mathrm{RH}} \sim \tau_{\mathrm{m}} \times\left(f_{\mathrm{RH}} / f_{\mathrm{m}}\right) \sim(0.7-0.8) \tau_{\mathrm{m}}$. In this case assuming no difference between the mass ratio of clusters with and without $\mathrm{RH}$, we find $\tau_{\mathrm{RH}} \sim 1.4-2.4 \mathrm{Gyr}$.

In general, we note that both different timescales $\left(\tau_{\mathrm{RH}} \lesssim \tau_{\mathrm{m}}\right.$; i.e. Scenario 2) and mass ratios (i.e. Scenario 1) are likely to govern the statistics of giant RHs.

This study deals with several limitations and is based on simplified assumptions:

(i) The observed fraction of merging clusters is derived by measuring the fraction of clusters with significant X-ray disturbance, this means that we are sensitive only to merger episodes with relevant mass accretion.

(ii) While observationally, the fraction of merging clusters is derived by measuring the morphological disturbances of clusters in the sample on a circular region of $\sim 1 \mathrm{Mpc}$ (diameter), the theoretical merging rate (FBMI10) and hence the merger fraction is derived by considering all the infalling halos within the virial radius $(\sim 2-3 \mathrm{Mpc})$ of the main clusters. However, it should be mentioned that numerical simulations allow us to argue that for $\xi \gtrsim 0.1$ the two rates should be comparable (see Sect. 4 and Tormen et al. 2004, for more details).

(iii) We assume that the X-ray disturbance that we measure in the X-rays is mainly caused by a binary merger event, specifically by that with larger mass ratio. Thus in deriving the expected merger fraction from theoretical fitting formulae, we attempt to select the values of $\xi_{\min }$ that matches the merger fraction assuming that mergers with smaller mass ratio do not play a role. In principle, however, in the timescale of the merger-induced disturbance, $\tau_{\mathrm{m}}$, clusters might experience multiple merger episodes with slightly lower mass ratio that can contribute to the morphological disturbance. We show that for typical merger timescale, constrained by simulations and the observed values of $\xi_{\min }$, the contribution of these slightly minor mergers is not expected to be relevant. Clearly, ad hoc simulations and follow-up studies are necessary to establish a more solid connection between mergers and X-ray disturbances.

It is currently thought that giant RHs are generated as a consequence of the acceleration of relativistic electrons by the MHD 
turbulence stirred up in the ICM by cluster-cluster mergers (e.g. Brunetti \& Jones 2014). In this framework scenario 1) discussed above implies that the timescale of the X-ray mergerinduced disturbances and that of the turbulent stirring of the ICM by cluster mergers in the central $1 \mathrm{Mpc}$ (diameter) region are similar. These timescales are shorter than the dynamical timescale of the merger, which is defined as the time interval between the moment when the centre of the less massive cluster first crosses the virial radius of the main one and the moment when the final system reaches a relaxed state. As a consequence, in this scenario RHs are not switched on at the beginning of the merger but after a time period that is necessary for the infalling subcluster to generate ICM turbulence in the central Mpc region ( $\sim .9$ Gyr; see Sect. 4.1). Since gravity drives mergers between galaxy clusters, it is expected that the turbulent energy budget should scale with the cluster thermal energy. As a consequence, very massive and merging systems should be the natural host of Mpc-scale RHs (e.g. Cassano \& Brunetti 2005; Vazza et al. 2006, 2011; Paul et al. 2011). In line with these expectations, we find $f_{\mathrm{RH}} \simeq 44-51 \%$ for clusters with $M_{500} \gtrsim 6 \times 10^{14} M_{\odot}$ at $0.2 \lesssim z \lesssim 0.33$ (see also Sommer \& Basu 2014; Cuciti et al. 2015). Also the mass-ratio may play a role because major mergers are more powerful events and have the potential to generate more turbulence in larger volumes. For instance, using a semi-analytic approach, Cassano \& Brunetti (2005) showed that, for a given cluster mass, the ratio between the turbulent energy and cluster thermal energy increases with increasing $\xi$, becoming smaller than $5 \%$ for $\xi<0.2$ (see also Fig. 3 in Cassano \& Brunetti 2005). This can explain the absence of RHs in clusters undergoing merger events with mass ratio $\xi<0.2$.

On the other hand, scenario 2) would imply that mergers drive turbulent re-acceleration of relativistic particles in the ICM on a timescale that is $\sim 0.7-0.8$ shorter than the timescale duration of the morphological disturbances in the X-rays. Lagrangian (SPH) simulations of two colliding idealized clusters have been used to study the time evolution of the RH emission during mergers (Donnert et al. 2013). These simulations predict shortly living RHs that are generated after the first core passage and fade within $\lesssim 1$ Gyr timescale. In fact, this timescale is shorter than that constrained assuming the scenario 2), however this can be due to the idealized set-up of the model and it is very likely that the lifetime of a RH is significantly larger in a cosmological contest. High resolution cosmological simulations also show an increase of the turbulence (both compressible and incompressible) and of the acceleration rate during major mergers (Miniati 2015). These simulations add also important information as they allow us to evaluate the ratio $\tau_{\mathrm{RH}} / \tau_{\mathrm{m}}$ under different assumptions about the ICM microphysics. This is an important point as it implies that statistical studies of the connection between RHs and merging rates combined with numerical simulations also have the potential to put fundamental constraints on the ICM microphysics and acceleration mechanisms (Miniati 2015; Brunetti 2016).

To conclude, while it is clear that massive and merging clusters are the natural hosts of giant RHs, the presence of merging clusters without RHs pose fundamental questions: is there a role of the merger mass ratio in the formation of a giant $\mathrm{RH}$ ? What is the lifetime of a RH with respect to the merger timescale? Is the $\mathrm{RH}$ lifetime tied by the microphysics of the ICM?

Our exploratory study has shown that meaningful values of the merger parameters can be derived combining the observed fraction of RHs and the theoretical merging rate in the $\Lambda$ CDM model. More specifically our results seem to suggest that the mass-ratio may play a role in the generation of RHs, however this result is not conclusive and we cannot conclude whether sce- nario 1) is favored with respect to scenario 2), or whether mass ratios and different timescales both play a role. An important step forwards to address the lifetime of $\mathrm{RHs}$ and the connection with mergers can be achieved by increasing the statistics of merging clusters without RHs. Our study is limited by current data that allow us to infer these constraints only in very massive clusters at relatively low redshifts. In fact in our study we use the currently most complete mass-selected sample of galaxy clusters with radio and X-ray information that, however, is limited to very massive $\left(M_{500} \gtrsim 6 \times 10^{14} M_{\odot}\right)$ and relatively nearby systems $(z \simeq 0.2-0.33)$. Based on energy arguments the occurrence of "radio quiet" merging clusters should increase at smaller masses (or at higher redshifts or small mass-ratio mergers; e.g. Cassano et al. 2006). Thus extending the samples of clusters at smaller masses (or at higher redshift) is necessary to obtain stronger constraints on the physical conditions necessary to generate RHs. This will be possible with the upcoming new generation of radio facilities, such as LOFAR, ASKAP, and MeerKAT up to SKA1 (e.g. Cassano et al. 2015). Future surveys also offer the possibility of exploring the frequency dependence of the occurrence of RHs in galaxy clusters. Current models predict the presence of RHs with ultra-steep radio spectra (USSRHs) especially in low massive (or high-z) galaxy clusters (e.g. Cassano et al. 2006; Brunetti et al. 2008). As a consequence, it is possible that some of the merging clusters without RHs actually host USSRHs and observations with LOFAR (and SKA1-LOW in the future) will be crucial to check this possibility.

Acknowledgements. We thank the anonymous referee for his/her valuable report. S.E. acknowledges support from ASI-INAF No. I/009/10/0 and NuSTARASI/INAF No. I/037/12/0. G.B. and R.C. acknowledge partial support from PRIN-INAF 2014. G.B. acknowledges support from the Alexander von Humboldt Foundation. We thank K. Dolag for useful discussion and V. Cuciti for providing the morphological parameters of five clusters (denoted with ${ }^{* * *}$ in Table A.1).

\section{References}

Bacchi, M., Feretti, L., Giovannini, G., \& Govoni, F. 2003, A\&A, 400, 465 Bardeau, S., Soucail, G., Kneib, J.-P., et al. 2007a, A\&A, 470, 449 Barrena, R., Boschin, W., Girardi, M., \& Spolaor, M. 2007b, A\&A, 467, 37 Barrena, R., Girardi, M., Boschin, W., De Grandi, S., \& Rossetti, M. 2014, MNRAS, 442, 2216

Bernardi, G., Venturi, T., Cassano, R., et al. 2016, MNRAS, 456, 1259 Basu, K. 2012, MNRAS, 421, 112

Böhringer, H., Pratt, G. W., Arnaud, M., et al. 2010, A\&A, 514, A32

Bonafede, A., Intema, H., Brüggen, M., et al. 2015, MNRAS, 454, 3391

Boschin, W., Girardi, M., Spolaor, M., \& Barrena, R. 2006, A\&A, 449, 461

Boylan-Kolchin, M., Springel, V., White, S. D. M., Jenkins, A., \& Lemson, G. 2009, MNRAS, 398, 1150

Brüggen, M., Bykov, A., Ryu, D., \& Röttgering, H. 2012, Space Sci. Rev., 166, 187

Brüggen, M., \& Vazza, F. 2015, in Magnetic Fields in Diffuse Media (Berlin: Springer-Verlag), 407, 599

Brunetti, G. 2016, Plasma Physics and Controlled Fusion, 58, 014011 Brunetti, G., \& Jones, T. W. 2014, Int. J. Mod. Phys. D, 23, 1430007

Brunetti, G., \& Lazarian, A. 2007, MNRAS, 378, 245

Brunetti G., Venturi, T., Dallacasa, D., et al. 2007, ApJ , 670, L5

Brunetti, G., Giacintucci, S., Cassano, R., et al. 2008, Nature, 455, 944

Brunetti G., Cassano, R. Dolag, K., \& Setti, G. 2009, A\&A, 507, 661

Buote, D. A., \& Tsai, J. C. 1995, ApJ, 452, 522

Cassano, R., \& Brunetti, G. 2005, MNRAS, 357, 1313

Cassano, R., Brunetti, G., \& Setti, G. 2006, MNRAS, 369, 1577

Cassano, R., Ettori, S., Giacintucci, S., et al. 2010, ApJ, 721, L82

Cassano, R., Ettori, S., Brunetti, G., et al. 2013, ApJ, 777, 141

Cassano, R., Bernardi, G., Brunetti, G., et al. 2015, Proc. of Conf. on Advancing Astrophysics with the Square Kilometre Array (AASKA14), 73

Conselice, C. J. 2014, ARA\&A, 52, 291

Conselice, C. J., Bershady, M. A., Dickinson, M., \& Papovich, C. 2003, AJ, 126, 1183 
Cuciti, V., Cassano, R., Brunetti, G., et al. 2015, A\&A, 580, A97

Dahle, H., Kaiser, N., Irgens, R. J., Lilje, P. B., \& Maddox, S. J. 2002, ApJS, 139,313

De Boni, C., Serra, A. L., Diaferio, A., Giocoli, C., \& Baldi, M. 2016, ApJ, 818 , 188

De Propris, R., Liske, J., Driver, S. P., Allen, P. D., \& Cross, N. J. G. 2005, AJ, 130,1516

Diaferio, A. 2015 ArXiv e-print [arXiv: 1502 .01195]

Donnert, J., Dolag, K., Brunetti, G., \& Cassano, R. 2013, MNRAS, 429, 3564

Duffy, A. R., Schaye, J., Kay, S. T., \& Dalla Vecchia, C. 2008, MNRAS, 390, L64

Ettori, S., Gastaldello, F., Leccardi, A., et al. 2010, A\&A, 524, A68

Fakhouri, O., \& Ma, C.-P. 2008, MNRAS, 386, 577

Fakhouri, O., Ma, C.-P., \& Boylan-Kolchin, M. 2010, MNRAS, 406, 2267

Feretti, L., Fusco-Femiano, R., Giovannini, G., \& Govoni, F. 2001, A\&A, 373 106

Feretti, L., Giovannini, G., Govoni, F., \& Murgia, M. 2012, A\&ARv, 20, 54 Giacintucci, S., Venturi, T., Cassano, R., Dallacasa, D., \& Brunetti, G. 2009, ApJ, 704, L54

Giacintucci, S., Dallacasa, D., Venturi, T., et al. 2011, A\&A, 534, A57

Giacintucci, S., Kale, R., Wik, D. R., Venturi, T., \& Markevitch, M. 2013, ApJ, 766,18

Giovannini, G., Feretti, L., Govoni, F., Murgia, M., \& Pizzo, R. 2006, Astron. Nachr. 327, 563

Giocoli, C., Moreno, J., Sheth, R. K., \& Tormen, G. 2007, MNRAS, 376, 977

Giocoli, C., Tormen, G., \& Sheth, R. K. 2012, MNRAS, 422, 185

Govoni, F., Enßlin, T. A., Feretti, L., \& Giovannini, G. 2001, A\&A, 369, 441

Govoni, F., Murgia, M., Giovannini, G., Vacca, V., \& Bonafede, A. 2011, A\&A, 529, A69

Guo, Q., \& White, S. D. M. 2008, MNRAS, 384, 2

Hopkins, P. F., Cox, T. J., Hernquist, L., et al. 2013, MNRAS, 430, 1901

Jian, H.-Y., Lin, L., \& Chiueh, T. 2012, ApJ, 754, 26

Kale, R., \& Parekh, V. 2016, MNRAS, 459, 2940

Kale, R., Venturi, T., Giacintucci, S., et al. 2013, A\&A, 557, A99

Kale, R., Venturi, T., Giacintucci, S., et al. 2015, A\&A, 579, A92

Kravtsov, A. V., \& Borgani, S. 2012, ARA\&A, 50, 353

Kulsrud, R. M., Cen, R., Ostriker, J. P., \& Ryu, D. 1997, ApJ, 480, 481

Landry, D., Bonamente, M., Giles, P., et al. 2013, MNRAS, 433, 2790

Lemze, D., Postman, M., Genel, S., et al. 2013, ApJ, 776, 91

Lotz, J. M., Primack, J., \& Madau, P. 2004, AJ, 128, 163

Lotz, J. M., Jonsson, P., Cox, T. J., et al. 2011, ApJ, 742, 103

Mahdavi, A., Hoekstra, H., Babul, A., Balam, D. D., \& Capak, P. L. 2007, ApJ, 668,806
Mantz, A. B., Allen, S. W., Morris, R. G., et al. 2015, MNRAS, 449, 199

Miniati, F. 2015, ApJ, 800, 60

Mohr, J. J., Fabricant, D. G., \& Geller, M. J. 1993, ApJ, 413, 492

McBride, J., Fakhouri, O., \& Ma, C.-P. 2009, MNRAS, 398, 1858

Moreno, J., Giocoli, C., \& Sheth, R. K. 2008, MNRAS, 391, 1729

Murgia, M., Govoni, F., Markevitch, M., et al. 2009, A\&A, 499, 679

Navarro, J. F., Frenk, C. S., \& White, S. D. M. 1997, ApJ, 490, 493

Norman, M. L., \& Bryan, G. L. 1999, The Radio Galaxy Messier 87, 530, 106

Okabe, N., \& Umetsu, K. 2008, PASJ, 60, 345

Okabe, N., Takada, M., Umetsu, K., Futamase, T., \& Smith, G. P. 2010, PASJ, 62,811

Parekh, V., van der Heyden, K., Ferrari, C., Angus, G., \& Holwerda, B. 2015, A\&A, 575, A127

Patton, D. R., Carlberg, R. G., Marzke, R. O., et al. 2000, ApJ, 536, 153

Paul, S., Iapichino, L., Miniati, F., Bagchi, J., \& Mannheim, K. 2011, ApJ, 726, 17

Planck Collaboration XX. 2014, A\&A, 571, A20

Planck Collaboration XXIX. 2014, A\&A, 571, A29

Poole, G. B., Fardal, M. A., Babul, A., et al. 2006, MNRAS, 373, 881

Reid, A. D., Hunstead, R. W., Lemonon, L., \& Pierre, M. M. 1999, MNRAS, 302,571

Ricker, P. M., \& Sarazin, C. L. 2001, ApJ, 561, 621

Rossetti, M., Eckert, D., Cavalleri, B. M., et al. 2011, A\&A, 532, A123

Santos, J.S., Rosati, P., Tozzi, P., Böhringer, H., Ettori, S., \& Bignamini, A. 2008, A\&A, 483, 35

Sommer, M. W., \& Basu, K. 2014, MNRAS, 437, 2163

Soucail, G. 2012, A\&A, 540, A61

Springel, V., White, S. D. M., Jenkins, A., et al. 2005, Nature, 435, 629

Subramanian, K., Shukurov, A., \& Haugen, N. E. L. 2006, MNRAS, 366, 1437

Tormen, G., Moscardini, L., \& Yoshida, N. 2004, MNRAS, 350, 1397

Vazza, F., Tormen, G., Cassano, R., Brunetti, G., \& Dolag, K. 2006, MNRAS, 369, L14

Vazza, F., Brunetti, G., Gheller, C., Brunino, R., \& Brüggen, M. 2011, A\&A, 529, A17

Venturi, T., Giacintucci, S., Brunetti, G., et al. 2007, A\&A, 463, 937

Venturi, T., Giacintucci, S., Dallacasa, D., et al. 2008, A\&A, 484, 327

Venturi, T., Giacintucci, G., Dallacasa, D., et al. 2011, MNRAS, 414, L65

van den Bosch, F. C. 2002, MNRAS, 331, 98

Wen, Z. L., \& Han, J. L. 2013, MNRAS, 436, 275

Yuan, Z. S., Han, J. L., \& Wen, Z. L. 2015, ApJ, 813, 77

Wittman, D., Dawson, W., \& Benson, B. 2014, MNRAS, 437, 3578

Ziparo, F., Braglia, F. G., Pierini, D., et al. 2012, MNRAS, 420, 2480 
R. Cassano et al.: Can giant radio halos probe the merging rate of galaxy clusters?

\section{Appendix A: Additional tables}

In Table A.1 we report the main properties of the 54 clusters belonging to our sample, specifically: Col. (1) cluster name; Col. (2) cluster redshift; Col. (3) $M_{500}$ from Planck Collaboration XXIX (2014); Col. (4) virial mass, $M_{\text {vir }}$ (see Sect. 2); Col. (5) information about the presence of diffuse radio emission; Col. (6) cluster dynamical status; Col. (7) mass ratio, when available.
The first two panels contain the 39 clusters with both radio and X-ray information (clusters with RHs are in the second panel); the third panel contains clusters with X-ray information; the three clusters in the forth panel are those without radio and X-ray information. In Table A.2 we report the morphological parameters derived for the 41 clusters with Chandra X-ray data (see Sect. 2): $P_{3} / P_{0}, w$ and $c$, with their inferior and superior values ( $\left[P_{3} / P_{0}-1 \sigma, P_{3} / P_{0}+1 \sigma\right]$, and so on for the others). 
Table A.1. Cluster properties.

\begin{tabular}{|c|c|c|c|c|c|c|}
\hline Cluster name & $z$ & $\begin{array}{c}M_{500} \\
{\left[10^{14} M_{\odot}\right]}\end{array}$ & $\begin{array}{c}M_{\mathrm{vir}} \\
{\left[10^{14} M_{\odot}\right]}\end{array}$ & Radio info & $\mathrm{X}$-ray info & Mass ratio $^{z}$ \\
\hline \multicolumn{7}{|c|}{ Clusters with radio and X-ray data } \\
\hline A2697 & 0.23 & 6.00 & 11.00 & $\mathrm{UL}^{a}$ & relaxed $^{1}$ & - \\
\hline A3088 & 0.25 & 6.71 & 12.25 & $\mathrm{UL}^{a}$ & relaxed $^{2}$ & - \\
\hline A2667 & 0.23 & 6.81 & 12.46 & $\mathrm{UL}^{a}$ & relaxed $^{3}$ & - \\
\hline RXJ0142.0+21 & 0.28 & 6.07 & 10.95 & $\mathrm{UL}^{b}$ & relaxed $^{2}$ & - \\
\hline A1423 & 0.21 & 6.09 & 11.08 & $\mathrm{UL}^{a}$ & relaxed $^{3}$ & - \\
\hline A1576 & 0.30 & 5.98 & 10.8 & $\mathrm{UL}^{b}$ & relaxed $^{2, *, * *}$ & 1:4-1:3 (Dahle et al. 2002) \\
\hline A2261 & 0.22 & 7.39 & 13.56 & $\mathrm{UL}^{b}$ & relaxed $^{3}$ & - \\
\hline A 2537 & 0.30 & 6.17 & 11.15 & $\mathrm{UL}^{a}$ & relaxed $^{3}$ & - \\
\hline S0780 & 0.24 & 7.71 & 14.22 & $\mathrm{MH}^{c}$ & relaxed $^{3}$ & - \\
\hline A 1835 & 0.25 & 8.46 & 15.53 & $\mathrm{MH}^{d}$ & relaxed $^{4}$ & - \\
\hline A2390 & 0.23 & 9.48 & 17.59 & $\mathrm{MH}^{e}$ & relaxed $^{3}$ & - \\
\hline RXCJ1504.1-02 & 0.22 & 6.98 & 12.80 & $\mathrm{MH}^{f}$ & relaxed $^{3}$ & - \\
\hline A3444 & 0.25 & 7.62 & 13.98 & $\mathrm{MH}^{c}$ & relaxed $^{4}$ & - \\
\hline A68 & 0.26 & 6.19 & 11.25 & $\mathrm{UL}^{c}$ & merger $^{4}$ & 1:6-1:5 (Okabe et al. 2010) \\
\hline A2631 & 0.28 & 6.97 & 12.75 & $\mathrm{UL}^{a}$ & merger $^{3}$ & 1:7-1:6 (Okabe et al. 2010) \\
\hline A781 & 0.30 & 6.36 & 11.50 & $\mathrm{UL}^{a, a x}$ & merger $^{3}$ & 1:3 (Wittman et al. 2014) \\
\hline A1763 & 0.23 & 8.29 & 15.25 & no $\mathrm{RH}^{a}$ & merger ${ }^{4}$ & 1:5-1:4 (Bardeau et al. 2007) \\
\hline PSZ1 G205.07-62.94 & 0.31 & 7.37 & 13.40 & no $\mathrm{RH}^{g}$ & merger ${ }^{1}$ & - \\
\hline A2744 & 0.31 & 9.56 & 17.48 & $\mathrm{RH}^{h}$ & merger $^{3}$ & 1:3 (Boschin et al. 2006) \\
\hline A209 & 0.21 & 8.17 & 15.02 & $\mathrm{RH}^{i}$ & merger $^{3}$ & - \\
\hline A2163 & 0.20 & 16.44 & 31.52 & $\mathrm{RH}^{l}$ & merger $^{3}$ & 1:3 (Soucail 2012) \\
\hline RXCJ2003.5-2323 & 0.32 & 7.48 & 13.57 & $\mathrm{RH}^{i}$ & merger $^{3}$ & - \\
\hline A520 & 0.20 & 7.06 & 13.06 & $\mathrm{RH}^{h}$ & merger $^{3}$ & 1:1 (Mahdavi et al. 07) \\
\hline A773 & 0.22 & 7.08 & 13.05 & $\mathrm{RH}^{h}$ & merger $^{3}$ & 1:4-1:10 (Barrera et al. 2007) \\
\hline A1758a & 0.28 & 7.99 & 14.68 & $\mathrm{RH}^{m}$ & merger $^{3}$ & 1:2 (Okabe et al. 2008) \\
\hline A1351 & 0.32 & 7.14 & 12.95 & $\mathrm{RH}^{n}$ & merger $^{4}$ & 1:5 (Barrera et al. 2014) \\
\hline A2219 & 0.23 & 11.01 & 20.40 & $\mathrm{RH}^{e}$ & merger $^{3}$ & \\
\hline A521 & 0.25 & 6.91 & 12.37 & $\mathrm{RH}^{o}$ & merger $^{3}$ & \\
\hline A697 & 0.28 & 11.48 & 21.37 & $\mathrm{RH}^{a}$ & merger $^{3}$ & \\
\hline PSZ1 G171.96-40.64 & 0.27 & 11.13 & 20.88 & $\mathrm{RH}^{p}$ & merger $^{1}$ & - \\
\hline A1300 & 0.31 & 8.83 & 16.15 & $\mathrm{RH}^{q}$ & merger $^{3}$ & 1:1 (Ziparo et al. 2011) \\
\hline RXC J1314.4-2515 & 0.24 & 6.15 & 11.20 & $\mathrm{RH}^{i}$ & merger ${ }^{1}$ & \\
\hline RXC J1514.9-1523 & 0.22 & 8.34 & 15.35 & $\mathrm{RH}^{f}$ & merger ${ }^{4}$ & - \\
\hline A1682 & 0.23 & 6.20 & 11.33 & $\mathrm{RH}^{a}$ & merger $^{3}$ & \\
\hline A1443 & 0.27 & 7.74 & 14.15 & $\mathrm{RH}^{s}$ & merger $^{* * *}$ & - \\
\hline Z5247 & 0.23 & 6.04 & 11.00 & $\mathrm{RH} ?^{c}$ & merger ${ }^{4}$ & 1:4 (Dahle et al. 2002) \\
\hline A2552 & 0.30 & 7.53 & 13.65 & $\mathrm{RH} ?^{c}$ & relaxed $?^{4, *}$ & - \\
\hline RXC J0510.7-0801 & 0.22 & 7.36 & 13.50 & $\mathrm{RH} ?^{c}$ & merger $\sqrt{ }$ & - \\
\hline A402 & 0.32 & 7.20 & 13.06 & $\mathrm{MH} ?^{r}$ & relaxed $^{* * *}$ & - \\
\hline \multicolumn{7}{|c|}{ Clusters with (only) X-ray data } \\
\hline A2895 & 0.23 & 6.15 & 11.22 & - & merger $^{\sqrt{ }}$ & - \\
\hline A2813 & 0.29 & 9.16 & 15.15 & - & merger $\sqrt{ }$ & - \\
\hline PSZ1G139.61+24 & 0.27 & 7.09 & 12.93 & - & relaxed $^{* * *}$ & - \\
\hline A2355 & 0.23 & 6.92 & 12.75 & - & merger $^{* * *}$ & - \\
\hline A1733 & 0.26 & 7.05 & 12.85 & - & merger ${ }^{* * *}$ & - \\
\hline MACS J2135-010 & 0.33 & 7.57 & 13.8 & - & merger $\sqrt{ }$ & - \\
\hline RXC J2051.1+0216 & 0.32 & 6.13 & 11.07 & - & merger $^{1}$ & - \\
\hline A2472 & 0.31 & 6.15 & 11.12 & - & merger $^{1}$ & - \\
\hline A56 & 0.30 & 6.20 & 11.25 & - & merger $^{1}$ & - \\
\hline A384 & 0.24 & 6.38 & 11.65 & - & relaxed $^{1}$ & - \\
\hline RXCJ1322.8+31 & 0.31 & 6.63 & 12.0 & - & relaxed $^{1}$ & - \\
\hline PSZ1 G019.12+3123 & 0.28 & 7.08 & 12.95 & - & merger $^{1}$ & - \\
\hline \multicolumn{7}{|c|}{ Clusters without radio and X-ray data } \\
\hline $\mathrm{ZwCl} 1028.8+1419^{*}$ & 0.31 & 6.11 & 11.05 & - & - & - \\
\hline A $3041^{*}$ & 0.23 & 6.12 & 11.24 & - & - & - \\
\hline $\mathrm{A} 220^{*}$ & 0.33 & 6.74 & 12.20 & - & - & - \\
\hline
\end{tabular}

Notes. Radio info.: ${ }^{(a)}$ Venturi et al. (2008); ${ }^{(b)}$ Kale et al. (2013); ${ }^{(c)}$ Kale et al. (2015); ${ }^{(d)}$ Murgia et al. (2009); ${ }^{(e)}$ Bacchi et al. (2003); ${ }^{(f)}$ Giacintucci et al. (2011); ${ }^{(g)}$ Ferrari et al. (priv. comm.); ${ }^{(h)}$ Govoni et al. (2001); ${ }^{(i)}$ Venturi et al. (2007); ${ }^{(I)}$ Feretti et al. $(2001)$; ${ }^{(m)}$ Giovannini et al. $(2006)$; ${ }^{(n)}$ Giacintucci et al. (2009); ${ }^{(o)}$ Brunetti et al. (2008; ${ }^{(p)}$ Giacintucci et al. (2013); ${ }^{(q)}$ Reid et al. (1999); ${ }^{(r)}$ Macario et al. (priv. comm.) ; ${ }^{(s)}$ Bonafede et al. (2015). X-ray info.: ${ }^{(1)}$ from XMM-Newton visual inspection; ${ }^{(2)}$ Cassano et al. (2013) ; (3) Cassano et al. (2010); (4) Cuciti et al. 2015 ; ( ${ }^{(\sqrt{ })}$ this paper . ${ }^{(a x)}$ Venturi et al. (2011) report on a possible RH in A781, however those observations were not conclusive (see also Govoni et al. (2011). ${ }^{(*)}$ Landry et al. (2013) classify these clusters as "unrelaxed" systems; ${ }^{(*)}$ Dahle et al. $(2002)$ report on some merger activity in this cluster. ${ }^{(z)}$ the mass ratio here is defined as $1 / \xi{ }^{\left({ }^{* * *}\right)} \mathrm{V}$. Cuciti, (priv. comm.). 
R. Cassano et al.: Can giant radio halos probe the merging rate of galaxy clusters?

Table A.2. Cluster morphological parameters.

\begin{tabular}{|c|c|c|c|}
\hline Cluster name & 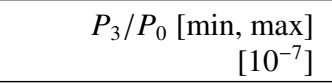 & $w[\min , \max ]$ & $c[\min , \max ]$ \\
\hline A3088 & $0.833[0.279,1.663]$ & $0.285[0.220,0.370]$ & $0.339[0.332,0.345]$ \\
\hline A2667 & $1.395[0.799,2.152]$ & $0.927[0.800,1.030]$ & $0.407[0.402,0.410]$ \\
\hline RXJ0142.0+21 & $6.625[3.655,10.350]$ & $0.738[0.650,0.910]$ & $0.186[0.180,0.191]$ \\
\hline A1423 & $1.413[0.656,3.880]$ & $0.562[0.460,0.760]$ & $0.331[0.323,0.342]$ \\
\hline A1576 & $5.950[3.661,11.071]$ & $1.271[0.940,1.590]$ & $0.235[0.226,0.241]$ \\
\hline A2261 & $1.026[0.513,1.673]$ & $0.495[0.430,0.570]$ & $0.334[0.330,0.337]$ \\
\hline A2537 & $0.351[0.165,1.208]$ & $0.561[0.460,0.660]$ & $0.278[0.273,0.282]$ \\
\hline S0780 & $0.480[0.243,0.801]$ & $0.827[0.760,0.880]$ & $0.473[0.470,0.476]$ \\
\hline A1835 & $0.459[0.317,0.576]$ & $0.996[0.952,1.032]$ & $0.487[0.485,0.488]$ \\
\hline A2390 & $0.694[0.520,0.933]$ & $1.171[1.120,1.200]$ & $0.305[0.303,0.306]$ \\
\hline RXCJ1504.1-02 & $0.148[0.086,0.221]$ & $0.459[0.430,0.490]$ & $0.624[0.622,0.626]$ \\
\hline A3444 & $0.434[0.256,0.650]$ & $0.745[0.683,0.806]$ & $0.465[0.461,0.467]$ \\
\hline A68 & $3.199[1.368,7.026]$ & $1.004[0.740,1.240]$ & $0.149[0.141,0.157]$ \\
\hline A2631 & $1.550[0.647,5.941]$ & $1.570[1.270,1.920]$ & $0.121[0.114,0.128]$ \\
\hline A781 & $3.143[0.711,11.880]$ & $6.374[5.830,6.770]$ & $0.111[0.103,0.118]$ \\
\hline A1763 & $1.222[0.480,2.509]$ & $1.885[1.686,2.039]$ & $0.139[0.135,0.143]$ \\
\hline A2744 & $11.050[7.995,14.070]$ & $2.637[2.490,2.760]$ & $0.101[0.098,0.103]$ \\
\hline A209 & $0.5 .185[0.136,1.465]$ & $1.321[1.150,1.460]$ & $0.176[0.170,0.181]$ \\
\hline A2163 & $14.850[13.770,16.120]$ & $5.970[5.890,6.020]$ & $0.116[0.115,0.118]$ \\
\hline RXCJ2003.5-2323 & $4.602[2.507,9.255]$ & $1.824[1.440,1.970]$ & $0.062[0.059,0.064]$ \\
\hline A520 & $5.259[4.779,5.588]$ & $10.050[10.030,10.110]$ & $0.0976[0.0971,0.0983]$ \\
\hline A773 & $1.445[0.659,2.705]$ & $2.403[2.220,2.530]$ & $0.184[0.179,0.188]$ \\
\hline A1758a & $2.515[1.492,3.697]$ & $8.217[8.070,8.320]$ & $0.109[0.106,0.111]$ \\
\hline A1351 & $3.506[1.900,7.398]$ & $4.272[3.872,4.527]$ & $0.083[0.079,0.088]$ \\
\hline A2219 & $1.681[1.228,2.068]$ & $2.127[2.070,2.190]$ & $0.134[0.133,0.136]$ \\
\hline A521 & $5.090[2.981,7.771]$ & $2.204[2.030,2.470]$ & $0.108[0.104,0.111]$ \\
\hline A697 & $1.668[0.790,3.919]$ & $0.731[0.580,0.890]$ & $0.153[0.149,0.157]$ \\
\hline A1300 & $6.847[4.079,12.880]$ & $4.442[4.230,4.640]$ & $0.191[0.185,0.197]$ \\
\hline RXC J1514.9-1523 & $1.411[0.491,2.995]$ & $1.301[1.063,1.429]$ & $0.064[0.062,0.066]$ \\
\hline A1682 & $15.320[8.342,24.490]$ & $2.054[1.820,2.390]$ & $0.126[0.119,0.132]$ \\
\hline A1443 & $12.890[6.644,21.020]$ & $3.530[3.138,3.817]$ & $0.109[0.101,0.115]$ \\
\hline Z5247 & $3.061[0.744,8.739]$ & $3.362[2.890,3.667]$ & $0.158[0.138,0.173]$ \\
\hline A2552 & $0.222[0.106,1.383]$ & $0.639[0.523,0.824]$ & $0.218[0.212,0.224]$ \\
\hline RXC J0510.7-0801 & $2.171[0.885,4.356]$ & $2.346[2.140,2.590]$ & $0.134[0.129,0.138]$ \\
\hline A402 & $1.350[0.793,3.169]$ & $1.249[1.109,1.399]$ & $0.323[0.315,0.331]$ \\
\hline A2895 & $4.851[2.929,7.732]$ & $4.271[4.020,4.440]$ & $0.161[0.155,0.167]$ \\
\hline A2813 & $1.230[0.396,3.239]$ & $0.311[0.300,0.550]$ & $0.172[0.168,0.176]$ \\
\hline PSZ1G139.61+24 & $0.194[0.074,0.965]$ & $1.348[1.175,1.526]$ & $0.362[0.358,0.369]$ \\
\hline A2355 & $7.495[4.403,12.331]$ & $4.879[4.458,5.066]$ & $0.075[0.071,0.080]$ \\
\hline A1733 & $0.299[0.629,7.244]$ & $4.219[3.738,4.642]$ & $0.134[0.121,0.142]$ \\
\hline MACS J2135-010 & $4.073[2.203,10.711]$ & $1.188[0.917,1.445]$ & $0.139[0.133,0.144]$ \\
\hline
\end{tabular}

